\title{
PEG mediated eco-friendly one pot sunthesis of benzylamine coumarin derivatives using multicomponent reactant
}

\author{
Denish J. Viradiya ${ }^{1,2}$, Vipul C. Kotadiya ${ }^{1}$, Mita D. Khunt ${ }^{1}$, Bharat H. Baria ${ }^{1,2}$, \\ Umed C. Bhoya ${ }^{1, *}$ \\ 1 Deparment of Chemistry, Saurashtra University, Rajkot-360005, Gujrat, India. \\ ${ }^{2}$ National Facility for Drug Discovery through New Chemical Entities Development and \\ Instrumentation Support to Small Pharma Enterprises, Saurashtra University Rajkot - 360005, India \\ *E-mail address: drucbhoya@gmail.com
}

\begin{abstract}
A green resourceful, eco-friendly and facile protocol was developed for the synthesis of benzylamine coumarin derivatives by the reaction of 4-hydroxy coumarin, secondary amine and aromatic aldehyde in the presence of $\mathrm{PEG}_{400}$ as a solvent as well as catalyst at room temperature. A wide range of functional groups were tolerated in the developed protocol. The structures of all the synthesized compounds were confirmed by ${ }^{1} \mathrm{H}$ NMR, IR, MASS and Elemental Analysis. The target molecules were obtained in good to excellent yield applying this method.
\end{abstract}

Keywords: One pot reaction; Benzylamine Coumarin derivative; Green synthesis; $\mathrm{PEG}_{400}$

\section{INTRODUCTION}

Multicomponent reactions (MCRs) are a dominant tool for atom efficient, time, costadvantageous and environmentally waste-free synthesis of complex building blocks of bioactive heterocycles [1]. In the synthesis of various chemicals, solvents and catalyst are accountable part of the chemical processes, thus selection of a proper solvent and catalyst should be done for synthesis of compounds. In last decades, emergence of "Green Chemistry" and "Green Solvents" concept and their applications increased greatly in field of chemistry [2]. During the past few years, the biologically compatible PEGs [3] were demonstrated to be a recyclable green reaction medium for various chemical reactions [4-6]. From the perspective of green chemistry, organic synthesis in PEG has received considerable attention because of its cost-effectiveness, easy availability and safe character. Furthermore, PEG and its monomethylethers have a low vapor pressure, nonflammable, nonvolatile and are available in high quantities at low prices. The application of PEG as a reaction medium is highly beneficial as the system remains neutral, which helps in maintaining a wide variety of functional groups unchanged that are either acid or base susceptible. For these reasons, PEG is considered to be an environmentally benign substitute to volatile organic solvents and a highly practical medium for the synthesis of heterocyclic compounds. 
In recent years, much interest has been in the synthesis of 3-benzyl substituted 4hydroxycoumarins owing to their fantastic application in various research fields including biological science and medicinal chemistry. 3-Benzyl substituted 4-hydroxycoumarin derivatives are components of numerous natural products like warfarin, phenprocoumon, coumatetralyl, carbochromen, bromadialone, etc. These compounds also exhibit a wide band of biological activities including antibacterial, anti-HIV [7], antiviral [8], anticoagulant [9], antioxidant [10] and anticancer activities [11]. The vast biological significance of the amino derivatives of 4-hydroxycoumarin inspired us to develop a novel protocol for the efficient synthesis of new benzylamine coumarin derivatives. A number of synthetic protocols have been applied for the synthesis of nitrogen containing coumarin derivatives [12-15]. Mannich reaction [16] is the best method to synthesize such type of compounds. The methods reported previously for the synthesis of benzylamine coumarin derivatives suffer from severe disadvantages such as longer reaction time, inadequate yields and use of expensive catalyt $[13,14]$. Thus, the development of environmentally benign, high-yielding, and fast synthesis of benzylamine coumarin derivatives still remains a desired goal in organic synthesis. Herein, we disclose a general, high yielding, without use of catalyt and green synthetic protocol for a wide variety of benzylamine coumarin derivatives starting from 4-hydroxycoumarin

\section{RESULT AND DISCUSSION}

Initially, we start the reaction by the use of benzaldehyde (1 mmol), 4hydroxycoumarin $(1 \mathrm{mmol})$, piperidine $(1 \mathrm{mmol})$ and water (as solvent). These meterials were employed as reactants for the model reaction to synthesize benzylamine coumarin derivatives in the absence of catalyst. But products were not formed, even after $24 \mathrm{~h}$ therefore, this reaction was further investigated. As per our knowledge this type of work has not been reported using green solvent.<smiles>O=c1cc(O)c2ccccc2o1</smiles>

1<smiles>[R][X]1ccc(C=O)cc1</smiles>

2

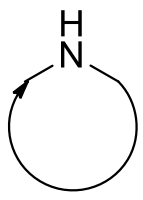

3<smiles>[R]c1cccc(C(c2c(O)c3ccccc3oc2=O)N2CCCCCCC2)c1</smiles>

$4(a-y)$

Scheme 1. synthesis of benzylamine coumarin derivatives.

Our main goal is to do the synthesis and explore the condition of reaction in poly ethylene glycol; therefore we use $\mathrm{PEG}_{200}, \mathrm{PEG}_{400}, \mathrm{PEG}_{600}$ and $\mathrm{PEG}_{800}$ as solvent. We performed the reaction at room temperature with above selected PEGs solvents and we got best results with $\mathrm{PEG}_{400}$ at $25-30{ }^{\circ} \mathrm{C}$ (Table 1). Further we carried out this reaction at different temperature in the presence of $\mathrm{PEG}_{400}$ (Table 2) but we cannot get more efficient result as compared to room temperature $\left(25-30^{\circ} \mathrm{C}\right)$. 
Table 1. Solvent effects on the three-component coupling reaction for the synthesis of benzylamine coumarin derivative $4 \mathrm{a}^{\mathrm{a}}$.

\begin{tabular}{cccc}
\hline Entry & Solvent & Time(h) & Yield\% \\
\hline $\mathbf{1}$ & $\mathrm{PEG}_{200}$ & 6 & 38 \\
$\mathbf{2}$ & $\mathrm{PEG}_{400}$ & 4 & 80 \\
$\mathbf{3}$ & $\mathrm{PEG}_{600}$ & 8 & 45 \\
$\mathbf{4}$ & $\mathrm{PEG}_{800}$ & 10 & 42 \\
\hline
\end{tabular}

${ }^{a}$ The reaction was carried out using 4-hydroxycoumarin $(1 \mathrm{mmol})$, piperidine $(1 \mathrm{mmol})$ and benzaldehyde( 1 $\mathrm{mmol})$ at room temperature $\left(25-30{ }^{\circ} \mathrm{C}\right)$.

Table 2. Influence of different temperature on the synthesis of benzylamine coumarin derivative $4 \mathrm{a}^{\mathrm{a}}$.

\begin{tabular}{cccc}
\hline Entry & Temperature $\left({ }^{\mathbf{0}} \mathbf{C}\right)$ & Time(h) & Yield \% \\
\hline $\mathbf{1}$ & $25-30$ & 4 & 80 \\
$\mathbf{2}$ & $60-65$ & 2 & 40 \\
$\mathbf{3}$ & $80-85$ & 4 & 60 \\
$\mathbf{4}$ & $100-110$ & 3 & 46
\end{tabular}

a The reaction was carried out using 4-hydroxycoumarin $(1 \mathrm{mmol})$, piperidine $(1 \mathrm{mmol})$ and benzaldehyde $(1$ $\mathrm{mmol}$ ) in presence of $\mathrm{PEG}_{400}$ for catalyst as well as solvent.

The quantity of the catalyst plays a critical role for the formation of the desired product. Then to extrapolate our research work, we were varying mole ratio of the $\mathrm{PEG}_{400}$ (Table 3) and we concluded that at $3 \mathrm{~mol} \%$, reaction was completed within 2-6 hrs only and excellent yield up to $80 \%$ was obtained.

Table 3. Optimization of catalyst $\left(\mathrm{PEG}_{400}\right)$ loading for the three component coupling reaction of4hydroxycoumarin, piperidine and benzaldehyde at $25-30^{\circ} \mathrm{C}$.

\begin{tabular}{ccc}
\hline Entry & Catalyst ratio (mol \%) & Yield \% \\
\hline $\mathbf{1}$ & 1 & 45 \\
$\mathbf{2}$ & 2 & 65 \\
$\mathbf{3}$ & 3 & 80 \\
$\mathbf{4}$ & 4 & 77 \\
\hline
\end{tabular}

These excellent preliminary results encouraged us to further explore the applicability of the catalyst $\left(3 \mathrm{~mol} \% \mathrm{PEG}_{400}\right)$ for the synthesis of benzylamine coumarin derivatives at room temperature (Scheme $1 \&$ Table 4). To study the scope and limitations of this protocol, we 
have employed a wide range of aromatic and hetero-aromatic aldehydes. Piperidine, morpholine and pyrolidine are the amine sources used to get the corresponding benzylamine coumarins. As mentioned in Table 4 the reaction proceeded smoothly with hetero-aromatic aldehydes, unsubstituted benzaldehyde, electron-withdrawing or electron-releasing substituted benzaldehydes.

Table 4. Synthesis of benzylamine coumarins derivatives ${ }^{\mathrm{a}}$.

\begin{tabular}{ccccc}
\hline Entry & $\mathbf{R}$ & Amine & Product & Yield (\%) \\
\hline $\mathbf{1}$ & $\mathrm{H}$ & Piperidine & $4 \mathrm{a}$ & 80 \\
$\mathbf{2}$ & $\mathrm{H}$ & Morpholine & $4 \mathrm{~b}$ & 75 \\
$\mathbf{3}$ & $\mathrm{H}$ & Pyrolidine & $4 \mathrm{c}$ & 79 \\
$\mathbf{4}$ & $3-\mathrm{OCH}_{3}$ & Piperidine & $4 \mathrm{~d}$ & 74 \\
$\mathbf{5}$ & $3-\mathrm{OCH}_{3}$ & Pyrolidine & $4 \mathrm{e}$ & 78 \\
$\mathbf{6}$ & $3-\mathrm{OCH}_{3}$ & Morpholine & $4 \mathrm{f}$ & 76 \\
$\mathbf{7}$ & $3-\mathrm{NO}_{2}$ & Morpholine & $4 \mathrm{~g}$ & 65 \\
$\mathbf{8}$ & $3-\mathrm{NO}_{2}$ & Piperidine & $4 \mathrm{~h}$ & 68 \\
$\mathbf{9}$ & $3-\mathrm{NO}_{2}$ & Pyrolidine & $4 \mathrm{i}$ & 71 \\
$\mathbf{1 0}$ & $4-\mathrm{pyridyl}$ & Piperidine & $4 \mathrm{j}$ & 76 \\
$\mathbf{1 1}$ & $4-p y r i d y l$ & Pyrolidine & $4 \mathrm{k}$ & 71 \\
$\mathbf{1 2}$ & $4-p y r i d y l$ & Morpholine & 41 & 73 \\
$\mathbf{1 3}$ & $4-\mathrm{Cl}_{14}$ & Pyrolidine & $4 \mathrm{~m}$ & 78 \\
$\mathbf{1 4}$ & $4-\mathrm{F}$ & Pyrolidine & $4 \mathrm{n}$ & 72
\end{tabular}

${ }^{\text {a }}$ The reaction was carried out using 4-hydroxycoumarin $(1 \mathrm{mmol})$, secondary amine $(1 \mathrm{mmol})$ and aromatic aldehyde $(1 \mathrm{mmol})$ in the presence of $\mathrm{PEG}_{400}(3 \mathrm{~mol} \%)$ at room temperature $\left(25-30{ }^{\circ} \mathrm{C}\right)$

From the above obtained results, we assume mechanistic activity of $\mathrm{PEG}_{400}$ as catalyst without any solvent media. The catalyst $\left(\mathrm{PEG}_{400}\right)$ plays an important role for the formation and stabilization of the imine intermediate. The catalyst $\left(\mathrm{PEG}_{400}\right)$ may induce 4hydroxycoumarin to act as the Mannich donor for the very fast formation of benzylamine coumarin derivatives.

\section{EXPERIMENTAL}

All of the Benzylamine coumarin derivatives have been characterized by ${ }^{1} \mathrm{H}-\mathrm{NMR}$, IR, Mass spectroscopy and CHNO analyzer. All the chemicals and solvents used for this work were obtained from E-Merck Ltd., Mumbai and S.D. Fine Chem. Ltd., Mumbai. Melting points of the synthesized compounds were determined in open capillary tubes and were uncorrected. IR absorption spectra were recorded on SIMADZU-FTIR-8400 series instrument, $\mathrm{KBr}$ diffuse reflectance, ${ }^{1} \mathrm{H}-\mathrm{NMR}$ spectra were recorded on a BRUKER Avance- 
III (400 MHz) spectrometer using DMSO- $d_{6}$ and $\mathrm{CDCl}_{3}$ as solvent and TMS (tetramethylsilane) as an internal standard. The ${ }^{1} \mathrm{H}$ chemical shifts were reported as parts per million (ppm) downfield from TMS ( $\mathrm{Me}_{4} \mathrm{Si}$ ). Mass spectra were determined in SHIMADZUGC-MS, Model No.QP-2010. ${ }^{1} \mathrm{H}-\mathrm{NMR}$ and IR spectra were consistent with the assigned structures. The elemental analysis (CHNS analysis) was done on a CHNS rapid analyzer. Purity of the compounds were checked by thin layer chromatography (TLC).

\section{1. General procedure for the synthesis of benzylamine coumarin derivatives; (4a-n)}

A mixture of 4-hydroxycoumarin $(1.0 \mathrm{mmol})$, aldehyde $(1.0 \mathrm{mmol})$ and secondary amine $(1.0 \mathrm{mmol})$ was stirred at room temperature in the presence of catalytic amount of $\mathrm{PEG}_{400}(3 \mathrm{~mol} \%)$. After the completion of the reaction (monitored by TLC), poured the reaction mass into crushed ice.

The product was isolated by filteration and crystallization by ethanol to give pure product. The isolated compounds were characterized by $\mathrm{mp}, \mathrm{IR},{ }^{1} \mathrm{H}$ NMR and elemental analysis $(\mathrm{C}, \mathrm{H}$ and $\mathrm{N})$.

\section{2. Analytical data for the synthesized compounds}

4-Hydroxy-3-(phenyl-piperidin-1-yl-methyl)-chromen-2-one; (4a)

M.P.: 181-183 ${ }^{\circ} \mathrm{C}$; IR (KBr): $3062,1672,1608,1497,1394,1242,1182,758 \mathrm{~cm}^{-1} ;{ }^{1} \mathrm{H}$ NMR (400 MHz, DMSO-d $\left.\mathrm{d}_{6}\right): \delta 1.49-1.57(\mathrm{~m}, 6 \mathrm{H}), 2.46(\mathrm{~s}, 1 \mathrm{H}), 2.94-2.98(\mathrm{~m}, 2 \mathrm{H}), 3.20(\mathrm{~s}, 1 \mathrm{H})$, $6.20(\mathrm{~s}, 1 \mathrm{H}), 7.04-7.23(\mathrm{~m}, 5 \mathrm{H}), 7.44(\mathrm{~m}, 2 \mathrm{H}), 7.76(\mathrm{~d}, 2 \mathrm{H})$; Anal. Calcd for $\mathrm{C}_{21} \mathrm{H}_{21} \mathrm{NO}_{3}: \mathrm{C}$ 75.20 , H 6.31 and N $4.18 \%$. Found: C 75.61, H 7.00 and N $4.77 \%$.

\section{4-Hydroxy-3-(morpholin-4-yl-phenyl-methyl)-chromen-2-one; (4b)}

M.P.: $168-170{ }^{\circ} \mathrm{C}$; IR (KBr): $3063,1673,1608,1555,1450,1395,1184,1106,759 \mathrm{~cm}^{-1} ;{ }^{1} \mathrm{H}$ NMR (400 MHz, $\left.\mathrm{CDCl}_{3}\right): \delta 2.32(\mathrm{~s}, 1 \mathrm{H}), 2.62(\mathrm{~s}, 2 \mathrm{H}), 3.30(\mathrm{~s}, 2 \mathrm{H}), 3.64-3.86(\mathrm{~m}, 4 \mathrm{H}), 7.22-$ $7.35(\mathrm{~m}, 5 \mathrm{H}), 7.48$ (br, $2 \mathrm{H}), 7.90-7.95(\mathrm{~m}, 2 \mathrm{H})$; Anal. Calcd for $\mathrm{C}_{20} \mathrm{H}_{19} \mathrm{NO}_{4}$ : C 71.20, H 5.68 and N 4.15\%. Found: C 71.30, H 5.51, and N $4.34 \%$.

\section{4-Hydroxy-3-(phenyl-pyrrolidin-1-yl-methyl)-chromen-2-one; (4c)}

M.P.: $171-172{ }^{\circ} \mathrm{C}$; IR (KBr): $3254,1666,1630,1498,1394,1219,1185,755 \mathrm{~cm}-1 ; 1 \mathrm{H}$ NMR (400 MHz, DMSO): $\delta$ 1.81-1.87 (m, 4H), 3.10 (br, 4H), 6.25(s, 1H), 6.92-7.05 (m, 5H), 7.29$7.34(\mathrm{~m}, 2 \mathrm{H}), 7.77(\mathrm{~d}, 2 \mathrm{H})$; Anal. calcd for $\mathrm{C}_{20} \mathrm{H}_{19} \mathrm{NO}_{3}$ : C $74.75, \mathrm{H} 5.96$ and $\mathrm{N} 4.36 \%$. Found: C 71.95, H 6.02, and N $4.40 \%$.

4-Hydroxy-3-[(3-methoxy-phenyl)-piperidin-1-yl-methyl]-chromen-2-one; (4d)

M.P.: $143{ }^{\circ} \mathrm{C}$; IR (KBr): 3030, 2850, 1690, 1630, 1529, 1400, 1275, 1180, 752cm-1; $1 \mathrm{H}$ NMR (400 MHz, $\left.\mathrm{CDCl}_{3}\right): \delta 1.62(\mathrm{~d}, 2 \mathrm{H}), 1.76-1.79(\mathrm{~m}, 4 \mathrm{H}), 3.22-3.26(\mathrm{~m}, 4 \mathrm{H}), 3.76-3.79$ (br, 3H) $6.17(\mathrm{~s}, 1 \mathrm{H}), 6.80(\mathrm{~d}, 2 \mathrm{H}), 7.21-7.32(\mathrm{~m}, 2 \mathrm{H}), 7.46-7.51(\mathrm{~m}, 1 \mathrm{H}), 8.10(\mathrm{~d}, 2 \mathrm{H}, J=6$ $\mathrm{Hz}$ ); Anal. calcd for $\mathrm{C}_{22} \mathrm{H}_{23} \mathrm{NO}_{4}$ : C 72.31, H 6.34, N $3.83 \%$. Found: C 72.28, H 6.30, and N $3.78 \%$.

\section{4-Hydroxy-3-[(3-methoxy-phenyl)-pyrrolidin-1-yl-methyl]-chromen-2-one; (4e)}

M.P.: $139-141{ }^{\circ} \mathrm{C}$; IR (KBr): $3035,1692,1624,1530,1454,1397,1180,753 \mathrm{~cm}^{-1} ;{ }^{1} \mathrm{H}$ NMR $\left(400 \mathrm{MHz} \mathrm{CDCl}_{3}\right): \delta 1.90-1.95(\mathrm{~m}, 4 \mathrm{H}), 3.33-3.40(\mathrm{~m}, 4 \mathrm{H}), 3.70(\mathrm{~s}, 3 \mathrm{H}), 6.01(\mathrm{~s}, 1 \mathrm{H}), 6.73$ $(\mathrm{d}, 2 \mathrm{H}), 7.11-7.25(\mathrm{~m}, 2 \mathrm{H}), 7.41-7.46(\mathrm{~m}, 2 \mathrm{H}), 8.05(\mathrm{~d}, 2 \mathrm{H})$; Anal. Calcd. for $\mathrm{C}_{21} \mathrm{H}_{21} \mathrm{NO}_{4}$ : C 71.78, H 6.02 and N $3.99 \%$. Found: C 71.60, H 6.25 and N $3.79 \%$.

4-Hydroxy-3-[(3-methoxy-phenyl)-morpholin-4-yl-methyl]-chromen-2-one; (4f)

M.P.: $146-148{ }^{\circ} \mathrm{C}$; IR (KBr): $3032,2850,1690,1622,1528,1394,1274,1179,750 \mathrm{~cm}^{-1} ;{ }^{1} \mathrm{H}$ 
NMR (400 MHz, $\left.\mathrm{CDCl}_{3}\right): \delta 3.02-3.05(\mathrm{~m}, 4 \mathrm{H}), 3.47(\mathrm{~s}, 3 \mathrm{H}), 3.65-3.70(\mathrm{~m}, 4 \mathrm{H}), 5.96(\mathrm{~s}, 1 \mathrm{H})$, $6.50(\mathrm{~d}, 2 \mathrm{H}), 6.88-7.01(\mathrm{~m}, 4 \mathrm{H}), 7.18-7.25(\mathrm{~m}, 2 \mathrm{H}), 7.72(\mathrm{~d}, 2 \mathrm{H})$; Anal. calcd for $\mathrm{C}_{21} \mathrm{H}_{21} \mathrm{NO}_{5}$ : C 68.65, H 5.76, N $3.81 \%$. Found: C 68.50, H 5.75, and N $3.91 \%$.

\section{4-Hydroxy-3-[morpholin-4-yl-(3-nitro-phenyl)-methyl]-chromen-2-one; (4g)}

M.P.: 190-192 ${ }^{\circ} \mathrm{C}$; IR (KBr): 3070, 2945, 1972, 1670, 1468, 1387, 1329, 985, $750 \mathrm{~cm}^{-1} ;{ }^{1} \mathrm{H}$ NMR (400 MHz, DMSO): $\delta 3.00-3.15(\mathrm{~m}, 4 \mathrm{H}), 3.78-3.80(\mathrm{~m}, 4 \mathrm{H}), 6.30(\mathrm{~s}, 1 \mathrm{H}), 7.08-7.16(\mathrm{~m}$, $2 \mathrm{H}), 7.25-7.40(\mathrm{~m}, 2 \mathrm{H}), 7.48-7.51(\mathrm{~m}, 2 \mathrm{H}), 7.81-7.94(\mathrm{~m}, 1 \mathrm{H}), 8.12(\mathrm{~s}, 1 \mathrm{H})$; Anal. calcd for $\mathrm{C}_{20} \mathrm{H}_{18} \mathrm{~N}_{2} \mathrm{O}_{6}$ : C 62.82, H 4.74 and $\mathrm{N} 7.33 \%$. Found: C 62.90, H 4.78 and N $7.37 \%$.

4-Hydroxy-3-[(3-nitro-phenyl)-piperidin-1-yl-methyl]-chromen-2-one; (4h)

M.P.: $190-192{ }^{\circ} \mathrm{C}$; IR (KBr): $3073,2950,1968,1640,1535,1399,1346,1068,760 \mathrm{~cm}^{-1} ;{ }^{1} \mathrm{H}$ NMR $\left(400 \mathrm{MHz}, \mathrm{CDCl}_{3}\right): \delta 1.46(\mathrm{br}, 1 \mathrm{H}), 1.75-1.90(\mathrm{~m}, 4 \mathrm{H}), 2.40-2.49(\mathrm{~m}, 2 \mathrm{H}), 2.76-2.98$ (m, 2H), 3.82-3.87 (br, 1H), $5.26(\mathrm{~s}, 1 \mathrm{H}), 7.23-7.35(\mathrm{~m}, 2 \mathrm{H}), 7.47-7.57(\mathrm{~m}, 2 \mathrm{H}), 7.99-8.04$ (br, $1 \mathrm{H}), 8.19(\mathrm{~d}, 2 \mathrm{H}), 8.43(\mathrm{~s}, 1 \mathrm{H})$; Anal. calcd for $\mathrm{C}_{21} \mathrm{H}_{20} \mathrm{~N}_{2} \mathrm{O}_{5}$ : C 66.31, H 5.30 and $\mathrm{N} 7.36$ \%. Found: C 66.40, H 5.31 and N $7.46 \%$.

\section{4-Hydroxy-3-[(3-nitro-phenyl)-pyrrolidin-1-yl-methyl]-chromen-2-one; (4i)}

M.P.: $187-190{ }^{\circ} \mathrm{C}$; IR (KBr): $3430,2945,1680,1594,1482,1395,1247,750 \mathrm{~cm}^{-1}$; ${ }^{1} \mathrm{H}$ NMR $\left(400 \mathrm{MHz}, \mathrm{CDCl}_{3}\right): \delta 1.47-1.59(\mathrm{~m}, 4 \mathrm{H}), 2.72-2.78(\mathrm{~m}, 4 \mathrm{H}), 5.06(\mathrm{~s}, 1 \mathrm{H}), 6.55-6.63(\mathrm{~m}, 2 \mathrm{H})$, 6.82-6.88 (m, 2H), 6.97-7.03 (m, 2H), $7.39(\mathrm{~d}, 1 \mathrm{H}), 7.56-7.59$ (br, 1H), 8.18 (br, 1H); Anal. calcd for $\mathrm{C}_{20} \mathrm{H}_{18} \mathrm{~N}_{2} \mathrm{O}_{5}$ : C 65.57, H 4.95 and N $7.65 \%$. Found: C 65.61, H 4.98 and N $7.67 \%$.

\section{4-Hydroxy-3-(piperidin-1-yl-pyridin-4-yl-methyl)-chromen-2-one; (4j)}

M.P.: $145-147{ }^{\circ} \mathrm{C}$; IR (KBr): $3250,2945,1698,1540,1462,1407,1280,1066,755 \mathrm{~cm}^{-1} ;{ }^{1} \mathrm{H}$ NMR (400 MHz, DMSO): $\delta$ 1.50-1.78 (m, 5H), $2.46(\mathrm{~s}, 1 \mathrm{H}), 2.94-2.98(\mathrm{~m}, 2 \mathrm{H}), 3.12(\mathrm{br}$, $2 \mathrm{H}), 5.38(\mathrm{~s}, 1 \mathrm{H}), 7.03-7.17(\mathrm{~m}, 2 \mathrm{H}), 7.37-7.47(\mathrm{~m}, 2 \mathrm{H}), 7.58-7.88(\mathrm{~m}, 2 \mathrm{H}), 8.22-8.52(\mathrm{~m}$, $2 \mathrm{H})$.; Anal. calcd for $\mathrm{C}_{21} \mathrm{H}_{24} \mathrm{~N}_{2} \mathrm{O}_{3}$ : C 71.57, H 6.86 and N $7.95 \%$. Found: C 71.62, H 6.71 and $\mathrm{N} 7.88 \%$.

\section{4-Hydroxy-3-(pyridin-4-yl-pyrroldin-1-yl-methyl)-chromen-2-one; (4k)}

M.P.: $148-150{ }^{\circ} \mathrm{C}$; IR (KBr): $3360,3035,1650,1524,1456,1203,1046,762 \mathrm{~cm}^{-1}$; ${ }^{1} \mathrm{H}$ NMR (400 MHz, DMSO): $\delta 1.92(\mathrm{br}, 4 \mathrm{H}), 3.20(\mathrm{br}, 4 \mathrm{H}), 5.46(\mathrm{~s}, 1 \mathrm{H}), 7.08-7.16(\mathrm{~m}, 3 \mathrm{H}), 7.39(\mathrm{t}$, $1 \mathrm{H}), 7.61(\mathrm{~d}, 2 \mathrm{H}, J=6 \mathrm{~Hz}), 7.82(\mathrm{~d}, 2 \mathrm{H}), 8.50(\mathrm{~s}, 1 \mathrm{H})$; Anal. calcd for $\mathrm{C}_{19} \mathrm{H}_{18} \mathrm{~N}_{2} \mathrm{O}_{3}$ : C 70.79 , H 5.63 and N 8.69 \%. Found: C 70.70, H 5.65 and N $8.65 \%$.

\section{4-Hydroxy-3-(morpholin-4-yl-pyridin-4-yl-methyl)-chromen-2-one; (4I)}

M.P.: $154-157{ }^{\circ} \mathrm{C}$; IR (KBr): $3065,2945,1694,1600,1398,1204,1027,750 \mathrm{~cm}^{-1} ;{ }^{1} \mathrm{H}$ NMR (400 MHz, DMSO): $\delta 3.10-3.16(\mathrm{~m}, 4 \mathrm{H}), 3.77-3.82(\mathrm{~m}, 4 \mathrm{H}), 6.30(\mathrm{~s}, 1 \mathrm{H}), 7.07-7.13(\mathrm{~m}, 2 \mathrm{H})$, 7.33-7.40 (m, 2H), $7.80(\mathrm{~d}, 2 \mathrm{H}), 8.28(\mathrm{~d}, 2 \mathrm{H})$; Anal. calcd for $\mathrm{C}_{19} \mathrm{H}_{18} \mathrm{~N}_{2} \mathrm{O}_{4}$ : C 67.44, H 5.36 and N $8.28 \%$. Found: C 67.45, H 5.36 and N $8.30 \%$.

\section{3-[(4-Chloro-phenyl)-pyrrolidin-1-yl-methyl]-4-hydroxy-chromen-2-one; (4m)}

M.P.: $166-168{ }^{\circ} \mathrm{C}$; IR (KBr): 3442, 2945, 1676, 1600, 1473, 1395, 1260, 749 $\mathrm{cm}^{-1}$; ${ }^{1} \mathrm{H}$ NMR $\left(400 \mathrm{MHz}, \mathrm{CDCl}_{3}\right): \delta 2.48(\mathrm{br}, 4 \mathrm{H}), 3.66(\mathrm{br}, 4 \mathrm{H}), 5.87(\mathrm{~s}, 1 \mathrm{H}), 7.54-7.66(\mathrm{~m}, 2 \mathrm{H}), 7.79-7.89$ $(\mathrm{m}, 2 \mathrm{H}), 8.20(\mathrm{~d}, 2 \mathrm{H}), 8.36(\mathrm{~d}, 2 \mathrm{H})$; Anal. calcd for $\mathrm{C}_{20} \mathrm{H}_{18} \mathrm{ClNO}_{3}: \mathrm{C} 67.51, \mathrm{H} 5.10$ and $\mathrm{N}$ $3.94 \%$. Found: C 67.60, H 5.10 and N $3.87 \%$.

3-[(4-Fluoro-phenyl)-pyrrolidin-1-yl-methyl]-4-hydroxy-chromen-2-one; (4n) M.P.: $156-159{ }^{\circ} \mathrm{C}$; IR (KBr): $3430,1607,1555,1458,1398,1299,755 \mathrm{~cm}^{-1}$; ${ }^{1} \mathrm{H}$ NMR (400 
$\left.\mathrm{MHz}, \mathrm{CDCl}_{3}\right): \delta$ 1.81-1.92 (m, 4H), 3.10-3.16 (m, 4H), $6.20(\mathrm{~s}, 1 \mathrm{H}), 6.74-6.80(\mathrm{~m}, 2 \mathrm{H}), 7.06-$ $7.15(\mathrm{~m}, 2 \mathrm{H}), 7.32-7.40(\mathrm{~m}, 2 \mathrm{H}), 7.76-7.82(\mathrm{~m}, 2 \mathrm{H}), 8.63(\mathrm{br}, 1 \mathrm{H})$; Anal. calcd for $\mathrm{C}_{20} \mathrm{H}_{18}$ $\mathrm{FNO}_{3}$ : C 70.78, H 5.35 and N $4.13 \%$. Found: C 70.86, H 5.30 and N $4.18 \%$.

\section{CONCLUSION}

In conclusion, a highly efficient and environmentally green methodology for the synthesis of benzylamine coumarin derivatives via one pot multi component reactions of 4hydroxycoumarin, aldehyde and secondary amine has been developed. The attractive features of this protocol are simple reaction procedure, easy product separation, purification and its high adaptability for the synthesis of a broad spectrum of benzylamine coumarin derivatives in good to excellent yields. To the best of our knowledge, this is the first report about the synthesis of benzylamine coumarin derivatives using green solvent $\left(\mathrm{PEG}_{400}\right)$ as a catalyst and mediator.

\section{ACKNOWLEDGEMENT}

We gratefully acknowledge financial support from U.G.C. and Department of Chemistry (DST-FIST Funded \& UGC-SAP Sponsored), Saurashtra University, Rajkot. Also thanks U.G.C, New Delhi, India for the grant of B.S.R. fellowship. Authors are also thankful to the National Facility for Drug Discovery (NFDD) Centre, Saurashtra University, Rajkot, for sample analysis.

\section{References}

[1] A. Domling, Chem. Rev. 17 (2006) 106

[2] J. Zhu Bienayme, H. Ed., Multicomponent Reactions, Wiley-VCH: Weinheim, 2005.

[3] D. Raman, M. Yus, Chem. Int. Ed. 44 (2005) 1602.

[4] M. Khunt, V. Kotadiya, D. Viradiya, B. Baria, U. Bhoya, International Letters of Chemistry, Physics and Astronomy 6 (2014) 61-68.

[5] M. Kidwai, D. Bhatnagar, N. Mishra, V. Bansal, Catal. Commun 9 (2008) 2547.

[6] D. Heldebrant, P. Jessop, J. Am. Chem. Soc. 125 (2003) 5600.

[7] L. Heiss, H. Gais, Tetrahedron Lett. 36 (1995) 3833.

[8] J. Xu, B. Liu, W. Wu, C. Qian, Q. Wu, X. Lin, J. Org. Chem. 71 (2006) 3991.

[9] S. Hesse, G. Kirsch, Tetrahedron Lett. 43 (2002) 1213.

[10] B. Lee, M. Clothier, F. Dutton, G. Conder, S. Johnson, Bioorg. Med. Chem. Lett. 8 (1998) 3317.

[11] J. Jung, Y. Jung, O. Park, Synth. Commun. 31 (2001) 1195.

[12] G. Melagraki, A. Afantitis, O. Igglessi-Markopoulou, A. Detsi, M. Koufaki, C. Kontogiorgis, D. Hadjipavlou-Litina, Eur. J. Med. Chem. 44 (2009) 3020.

[13] J. Jung, J. Lee, S. Oh, J. Lee, O. Park, Bioorg. Med. Chem. Lett. 14 (2004) 5527. 
[14] E. Ghabraiea, M. Bararjaniana, S. Balalaie, F. Rominger, H. Bijanzadeh, Helv. Chim. Acta 94 (2011) 1440.

[15] N. Robertson, P. Link J.Am. Chem. Soc. 75 (1953) 1883.

[16] A. Kumar, M. Gupta, M. Kumar, Tetrahedron Lett. 52 (2011) 4521.

[17] N. Vukovic, S. Sukdolak, S. Solujic, N. Niciforovic, Arch. Pharmacol. Res. 33 (2010) 5.

[18] G. Zhao, T. Jiang, H. Gao, B. Han, J. Huang, D. Sun, Green Chem. 6 (2004) 75. 